\title{
Comparing random coefficient autoregressive model with and without autocorrelated errors by Bayesian analysis
}

\author{
Autcha Araveeporn \\ Department of Statistics, Faculty of Science, King Mongkut's Institute of Technology Ladkrabang, Bangkok, 10520, \\ Thailand \\ E-mail: kaautcha@hotmail.com
}

\begin{abstract}
We proposed a Bayesian analysis for estimating an unknown parameter in a Random Coefficient Autoregressive (RCA) model and its AutoRegressive (AR) process errors. We called this model an RCA model with autocorrelated errors (RCA-AR). A Markov Chain Monte Carlo (MCMC) method was used to generate samples from a posterior distribution which, after having been averaged, gave the estimated value of the unknown parameter. We used a Gibbs sampling algorithm in our MCMC calculation. To compare the performances of the RCA and the RCA-AR models, a simulation was performed with a set of test data and then the mean square errors obtained were used to indicate their performance. The result was that the RCA-AR model worked better than the RCA model in every case. Lastly, we tried both models with real data. They were used to estimate a series of monthly averages of the Stock Exchange of Thailand (SET) index. The result was that the RCA-AR still worked better than the RCA model, similar to the simulation of test data.
\end{abstract}

Keywords: Autoregressive process, Bayesian analysis, Gibbs sampling, Markov Chain Monte Carlo, random coefficient autoregressive

\section{Introduction}

In finance, it is very common to see data in the form of time series that often show in the term of volatility when volume suddenly changes on the economics system, especially if the data are collected over a long period of time. Researchers have long been interested in modeling time series data to extract a time dependent feature of conditional variance or volatility. There are several volatility models for financial time series such as an AutoRegressive Conditional Heteroscedastic (ARCH) model introduced by Engle [1]. After it was introduced, the ARCH model has been extended by Bollerslev [2], creating a Generalized ARCH (GARCH) model. The GARCH model allows incorporation of past observations and past volatility into the model.

There are volatility models that use random coefficients such as the Conditional Heteroscedastic Au- toregressive Moving Average (CHARMA) model by Tsay [2]. This CHARMA model uses the coefficients of an Autoregressive Moving Average (ARMA) model and the second-order properties of the ARCH model. A special variant of the CHARMA model with a reduced number of parameters called a Random Coefficient Autoregressive (RCA) model was studied by Nicholls and Quinn [3].

To estimate the parameters in an RCA model, Nicholls and Quinn [3] employed a least square method and the maximum likelihood method. Hwang and Basawa [4] used conditional least square and weighted least square estimators, then Chandra and Taniguchi [5] used a conditional least square and classical moment estimators to estimate unknown parameters in a RCA model. Wang and Ghosh [6] used a Bayesian approach to estimate the first order parameters of the RCA model when the time series data do not have a constant variance and highly volatile periods. 
Parameter estimation of time series models is also difficult to estimate and might mislead the results because the error terms are the systematic relationships, the so-called autocorrelated errors. Several autoregressive models have appeared in the literature. Haggan and Ozaki [7] modeled nonlinear vibration by using an amplitude-dependent autoregressive time series model called an Exponential Autoregressive (EXPAR) model. Tong [8] introduced a Threshold AutoRegressive (TAR) model for nonlinear time series. This problem is most noticeable in time series data, since the each of the time series data element has a correlation depended on period of time. However, we thought that these errors affected the estimation of unknown parameters, so we applied an autoregressive process on the error terms.

We organize this paper as follows: Section 2 describes the RCA model and the autoregressive process; Section 3 describes the Bayesian approach to estimating parameters; Section 4 shows the prior distribution of the parameters; and Section 5 presents the Gibbs sampling algorithm implementation in the WinBUGS program. We apply our proposed method to simulated data and real data of monthly averaged SET indexes in Sections 6 and 7. Finally, in Section 8, we conclude with the results from simulated and real data.

\section{The RCA model with autocorrelated errors}

Consider the following Random Coefficient Autoregressive (RCA) model of order $\mathrm{p}$ called $\mathrm{RCA}(\mathrm{p})$ that is given by

$$
x_{t}=\alpha+\sum_{i=1}^{p} \beta_{t i} x_{t-i}+\varepsilon_{t}, t=2,3, \ldots, n .
$$

Wang and Ghosh [6] suggested

$$
\underline{\beta}_{t}=\underline{\mu}_{\beta}+\sum_{\beta}^{1 / 2} \underline{u}_{t}
$$

where $\alpha$ is the scalar constant, $\underline{\beta}_{t}=\left(\beta_{t 1}, \ldots, \beta_{t p}\right)^{\top}$, and $\underline{\mu}_{\beta}=\left(\mu_{\beta 1}, \ldots, \mu_{\beta p}\right)^{\top}$. It is assumed that $\varepsilon_{t}$ 's and $\underline{u}$ 's are the sequence of iid (independent and identically distributed random variables) from a distribution with mean zero and unit variance. The modelling of time series data is a good performance when the errors are shown the randomization, but the most of error terms are correlated between members of a series of number arrange in time. In this case, we focus the errors of time series data which refers to correlation in a class AutoRegressive (AR) process following,

$$
\varepsilon_{t}=\sum_{j=1}^{q} \rho_{j} \varepsilon_{t-j}+e_{t}, t=2,3, \ldots, n,
$$

where $e_{t}$ is assumed a white noise that are independently and identically distributed.

The RCA model based on autocorrelated errors was defined by $\operatorname{RCA}(\mathrm{p})-\mathrm{AR}(\mathrm{q})$, we will write as

$$
\begin{aligned}
& x_{t}=\alpha+\sum_{i=1}^{p} \beta_{t i} x_{t-i}+\sum_{j=1}^{q} \rho_{j} \varepsilon_{t-j}+e_{t}, \\
& t=2,3, \ldots, n . \\
& \underline{\beta}_{t}=\underline{\mu}_{\beta}+\sum_{\beta}^{1 / 2} \underline{u}_{t},
\end{aligned}
$$

where $\rho=\left(\rho_{1}, \ldots, \rho_{q}\right)^{\top}$ is the $q \times 1$ vector of constant, and $e_{t}$ 's are the errors of AR(q) model.

In this paper, we focus on the simplicity case study of first order on RCA(1) model as the RCA model (Wang and Ghosh [6]) and conditional heteroscedastic autoregressive model (CHARN) model that studied to model the foreign exchange rate (Bossaerts, Hardle, and Hafner [9]).

$$
\begin{aligned}
& x_{t}=\alpha+\beta_{t} x_{t-1}+\varepsilon_{t}, t=2,3, \ldots, n . \\
& \beta_{t}=\mu_{\beta}+\sigma_{\beta} u_{t} .
\end{aligned}
$$

Next, we obtain

$$
\hat{\varepsilon}_{t}=x_{t}-\hat{\alpha}+\hat{\mu}_{\beta} x_{t-1}, t=2,3, \ldots, n .
$$

In this case, RCA(1) model with autocorrelated errors in the first order is denoted by $\mathrm{RCA}(1)-\mathrm{AR}(1)$ model that can be shown as

$$
x_{t}=\hat{\alpha}+\hat{\mu}_{\beta} x_{t-1}+\rho \hat{\varepsilon}_{t-1}+e_{t}, t=2,3, \ldots, n .
$$

where $x_{t}$ 's are iid random variables with mean $\mu_{\beta}$, and variance $\sigma_{\beta}^{2}, \varepsilon_{t}$ 's are iid random variables with mean 0 and variance $\sigma_{\varepsilon}^{2}$, and $\beta_{t}$ 's and $\varepsilon_{t}$ 's are independent.

For the parameter estimation of RCA(1) model, it is seen from Eq. (4) that consisted of the intercept term $\alpha$, the mean $\mu_{\beta}$, variance $\sigma_{\beta}^{2}$ of the coefficient $\beta_{t}$, the variance $\sigma_{\varepsilon}^{2}$ of the $\varepsilon_{t}$, or defined as $\theta_{1}=\left(\alpha, \mu_{\beta}, \sigma_{\beta}^{2}, \sigma_{\varepsilon}^{2}\right)^{\top}$. The $\operatorname{RCA}(1)$-AR(1) model gives the parameter as the variance $\sigma_{e}^{2}$ of the $e_{t}$, and the coefficient of AR process, or defined as $\theta_{2}=\left(\rho, \sigma_{e}^{2}\right)^{\top}$. 


\section{Parameter estimation}

\subsection{RCA(1) model}

In Bayesian estimation for the RCA(1) model, we propose a three-level hierarchical model. At the first level is the conditional distribution of the data $x_{t}$ 's given the observed random variables $x_{t-1}$, coefficient $\alpha, \beta_{t}$, and $\sigma_{\varepsilon}^{2}$. The second level consists of the conditional distribution $\beta_{t}$ given the parameter $\mu_{\beta}$ and $\sigma_{\beta}^{2}$. Finally the last level shows the prior distribution of $\theta_{1}=\left(\alpha, \mu_{\beta}, \sigma_{\beta}^{2}, \sigma_{\varepsilon}^{2}\right)^{\top}$. Consequently, given the sample variables $x_{1}, x_{2}, \ldots, x_{n}$, we are able to express the RCA(1) model in the following hierarchical structure,

$$
\begin{aligned}
& x_{t} \mid x_{t-1}, \alpha, \beta_{t}, \sigma_{\varepsilon}^{2} \sim N\left(\alpha+\beta_{t} x_{t-1}, \sigma_{\varepsilon}^{2}\right), \\
& \beta_{t} \mid \mu_{\beta}, \sigma_{\beta}^{2} \sim N\left(\mu_{\beta}, \sigma_{\beta}^{2}\right), \\
& \left(\alpha, \mu_{\beta}, \sigma_{\beta}^{2}, \sigma_{\varepsilon}^{2}\right) \sim p\left(\alpha, \mu_{\beta}, \sigma_{\beta}^{2}, \sigma_{\varepsilon}^{2}\right),
\end{aligned}
$$

where $p(\cdot)$ is the prior density of $\theta_{1}$ which reflects our prior about the unknown parameters. Following Eq. (7), we can express the likelihood function of $\theta_{1}$ as,

$$
\begin{aligned}
& L\left(\alpha, \mu_{\beta}, \sigma_{\beta}^{2}, \sigma_{\varepsilon}^{2} \mid x_{1}, x_{2}, \ldots, x_{n}, \varepsilon_{1}, \ldots, \varepsilon_{n}\right) \\
& =\phi\left(x_{1} ; \alpha, \sigma_{\varepsilon}\right) \prod_{i=2}^{n} \phi\left(x_{t} ; \alpha+\beta_{t} x_{t-1},\right. \\
& \left.\quad \sqrt{\sigma}_{\varepsilon}^{2}+\sigma_{\beta}^{2} x_{t-1}^{2}\right),
\end{aligned}
$$

where $\phi(x ; \mu, \sigma)$ denotes the density function of a normal distribution with mean $\mu$ and standard deviation $\sigma$. Therefore, the joint posterior density of the parameters is given by

$$
\begin{aligned}
& f\left(\theta_{1} \mid x_{1}, x_{2}, \ldots, x_{n}\right) \propto L\left(\theta_{1} \mid x_{1}, x_{2}, \ldots, x_{n}\right) \\
& , p\left(\theta_{1}\right)
\end{aligned}
$$

where $p(\theta)$ is a prior density of $\theta$. From the hierarchical structure in Eq. (8), the joint posterior density can be written as

$$
\begin{aligned}
& f\left(\mu_{\beta} \mid x_{1}, x_{2}, \ldots, x_{n}\right) \propto \\
& \quad \int f\left(\alpha, \mu_{\beta}, \sigma_{\beta}^{2}, \sigma_{\varepsilon}^{2} \mid x_{1}, x_{2}, \ldots, x_{n}\right) d \alpha d \sigma_{\beta}^{2} d \sigma_{\varepsilon}^{2} .
\end{aligned}
$$

To deal with the complicated likelihood function, we use the Markov Chain Monte Carlo (MCMC) method to generate samples from the posterior distribution of $\theta_{1}=\left(\alpha, \mu_{\beta}, \sigma_{\beta}^{2}, \sigma_{\varepsilon}^{2}\right)^{\top}$. We will carry out the Gibbs sampler see [10], a widely used MCMC method, to obtain the parameter from the posterior distribution using the software WinBUGS.

\section{2. $R C A(1)-A R(1)$ model}

At this point, we only know the Bayesian estimation of data analysis on RCA(1) model, and we get $\hat{\theta}_{1}=\left(\hat{\alpha}, \hat{\mu}_{\beta}, \hat{\sigma}_{\beta}^{2}, \hat{\sigma}_{\varepsilon}^{2}\right)^{\top}$. We propose the hierarchical model, for which the conditional distribution of the data $x_{t}$ 's given the unobserved random variables $x_{t-1}$, coefficient $\rho$ and $\sigma_{e}^{2}$, and the prior distribution of $\theta_{2}=\left(\rho, \sigma_{e}^{2}\right)^{\top}$. Therefore the sample variables $x_{1}, x_{2}, \ldots, x_{n}$, we can make the RCA(1)-AR(1) model in the following hierarchical structure;

$$
\begin{aligned}
& x_{t} \mid x_{t-1}, \rho, \sigma_{e}^{2} \sim N\left(\hat{\alpha}+\hat{\mu}_{\beta} x_{t-1}+\rho \hat{\varepsilon}_{t-1}, \sigma_{e}^{2}\right),(9) \\
& \left(\rho, \sigma_{e}^{2}\right) \sim p\left(\rho, \sigma_{e}^{2}\right),
\end{aligned}
$$

where $p(\cdot)$ is the prior density of $\theta_{2}$ which unknown parameter. Following Eq. (9), we can express the likelihood function of $\theta_{2}$ as

$$
\begin{aligned}
& L\left(\rho, \sigma_{e}^{2} \mid x_{2}, \ldots, x_{n}, e_{2}, \ldots, \varepsilon_{n}\right) \\
& \quad=\phi\left(x_{2} ; \rho, \sigma_{e}^{2}\right) \prod_{i=3}^{n} \phi\left(x_{t} ; \hat{\alpha}+\hat{\mu}_{\beta} x_{t-1}+\right. \\
& \left.\quad \rho \hat{\varepsilon}_{t-1}, \sqrt{\sigma}_{e}^{2}+\hat{\mu}_{\beta}^{2} \sigma_{\beta}^{2}+\rho^{2} \hat{\sigma}_{\varepsilon}^{2}\right),
\end{aligned}
$$

Hence, under certain RCA(1) model, the Markov Chain Monte Carlo (MCMC) methods can be thought of as approximate posterior distribution of $\theta_{2}=$ $\left(\rho, \sigma_{e}^{2}\right)^{\top}$.

\section{Prior distribution}

Bayesian analysis combines prior information about model parameters with information from observed data, thereby generating a posterior distribution. Bayesian analysis requires prior distribution which is difficult to specify analytically for the model considered.

For the parameter estimation of RCA(1) model, the prior distribution of $\theta_{1}=\left(\alpha, \mu_{\beta}, \sigma_{\beta}^{2}, \sigma_{\varepsilon}^{2}\right)^{\top}$ are considered to be a continuous random variable in the set of real numbers following the normal distribution, and $\sigma_{\beta}^{2}, \sigma_{\varepsilon}^{2}$ are denoted in the set of real positive numbers following the inverse gamma distribution.

An RCA(1)-AR(1) model, the prior distribution of $\theta_{2}=\left(\rho, \sigma_{e}^{2}\right)^{\top}$ is defined that $\rho$ is the set of real number between interval $[-1,1]$ as a uniform distribution, and $\sigma_{e}^{2}$ is the set of real positive numbers following the inverse gamma distribution. 


\section{Posterior distribution via gibbs sampling}

To manage Bayesian analysis for $\mathrm{RCA}(1)$ and $\mathrm{RCA}(1)-\mathrm{AR}(1)$ models, we are interested in the properties of the density of $\theta_{1}=\left(\alpha, \mu_{\beta}, \sigma_{\beta}^{2}, \sigma_{\varepsilon}^{2}\right)^{\top}$ and $\theta_{2}=\left(\rho, \sigma_{e}^{2}\right)^{\top}$. Deriving the joint posterior density for $\theta_{1}$ amounts to integrate out the unobserved coefficients $\alpha, \mu_{\beta}, \sigma_{\beta}^{2}$, and $\sigma_{\varepsilon}^{2}$. The joint posterior density for $\theta_{2}$ is integrated for coefficients $\rho$ and $\sigma_{e}^{2}$. However it is difficult to perform both analytically and numerically when the observed data are not normally distributed. We can perform the likelihood function to obtain posterior estimator.

MCMC methods consist of algorithms to construct a Markov chain of the parameters such that its stationary distribution is our distribution of interest, i.e. the posterior distribution of the parameter of interest. That means, under some regularity conditions the realization of this Markov chain can be thought of as points sample from the posterior distribution.

We use the Gibbs sampler [11] which is the most popular MCMC method, to obtain dependent samples from the posterior distribution. Specifically, we derive the condition densities each parameters of $\theta_{1}$ and $\theta_{2}$.

\section{1. $R C A(1)$ model}

The condition densities of parameter in $\mathrm{RCA}(1)$ model are $f\left(\alpha \mid \mu_{\beta}, \sigma_{\beta}^{2}, \sigma_{\varepsilon}^{2}, \underline{x}\right), f\left(\mu_{\beta} \mid \alpha, \sigma_{\beta}^{2}, \sigma_{\varepsilon}^{2}, \underline{x}\right)$, $f\left(\sigma_{\beta}^{2} \mid \alpha, \mu_{\beta}, \sigma_{\varepsilon}^{2}, \underline{x}\right)$, and $f\left(\sigma_{\varepsilon}^{2} \mid \alpha, \mu_{\beta}, \sigma_{\beta}^{2}, \underline{x}\right)$, as the full conditional densities of $\alpha, \mu_{\beta}, \sigma_{\beta}^{2}$, and $\sigma_{\varepsilon}^{2}$, respectively, based on model Eq. (7).

The Gibbs sampling algorithm is:

1. Initialize $\alpha^{(0)}, \mu_{\beta}^{(0)}, \sigma_{\beta}^{2(0)}$, and $\sigma_{\varepsilon}^{2(0)}$, for $k=$ $1,2, \ldots, m+M$

2. Draw $\alpha^{(k)}$ from $f\left(\alpha \mid \mu_{\beta}^{(k-1)}, \sigma_{\beta}^{2(k-1)}, \sigma_{\varepsilon}^{2(k-1)}\right.$, $\underline{x})$,

$$
\begin{aligned}
& \text { Draw } \mu_{\beta}^{(k)} \text { from } f\left(\mu_{\beta} \mid \alpha^{(k)}, \sigma_{\beta}^{2(k-1)}, \sigma_{\varepsilon}^{2(k-1)}, \underline{x}\right) \text {, } \\
& \text { Draw } \sigma_{\beta}^{2(k)} \text { from } f\left(\sigma_{\beta}^{2} \mid \alpha^{(k)}, \mu_{\beta}^{(k)}, \sigma_{\varepsilon}^{2(k-1)}, \underline{x}\right), \\
& \text { Draw } \sigma_{\varepsilon}^{2(k)} \text { from } f\left(\sigma_{\varepsilon}^{2} \mid \alpha^{(k)}, \mu_{\beta}^{(k)}, \sigma_{\beta}^{2(k)}, \underline{x}\right),
\end{aligned}
$$

where $m$ is burn-in and $M$ is the number of samples generated after burn-in. Repeating the above sampling steps, we obtain a discrete-time Markov chain $\left\{\left(\alpha^{(k)}, \mu_{\beta}^{(k)}, \sigma_{\beta}^{2(k)}, \sigma_{\varepsilon}^{2(k)}\right) ; k=1,2, \ldots\right\}$ whose stationary distribution is the joint posterior density of the parameters.

Gibbs sampling algorithm is proposed above using a software package known as WinBUGS. In WinBUGS, a Markov chain of parameters of $\alpha, \mu_{\beta}, \sigma_{\beta}^{2}$, and $\sigma_{\varepsilon}^{2}$, are constructed by computing the mean sampling from the joint posterior density as standard distribution. We can perform this procedure using a freeware WinBUGS [12].

The MCMC samples of $\theta_{1}=\left(\alpha, \mu_{\beta}, \sigma_{\beta}^{2}, \sigma_{\varepsilon}^{2}\right)^{\top}$ obtain via WinBUGS which is computed to approximate posterior summaries of the parameters as the posterior estimation of $\theta$. In particular, we use the posterior mean as point estimates of $\theta$.

\section{2. $R C A(1)-A R(1)$ model}

The condition densities of parameter in $\mathrm{RCA}(1)$ $\mathrm{AR}(1)$ model are $f\left(\rho \mid \sigma_{e}^{2}, \underline{x}\right)$, and $f\left(\sigma_{e}^{2} \mid \rho, \underline{x}\right)$, as the full conditional densities of $\rho$ and $\sigma_{e}^{2}$ based on model Eq. (10).

The Gibbs sampling algorithm is:

1. Initialize $\rho^{(0)}$, and $\sigma_{e}^{2(0)}$, for $k=1,2, \ldots, m+$ M

2. Draw $\rho^{(k)}$ from $f\left(\rho \mid \sigma_{e}^{2(k-1)}, x\right)$, Draw $\sigma_{e}^{2(k)}$ from $f\left(\sigma_{e}^{2} \mid \rho^{(k)}, x\right)$,

where $m$ is burn-in and $M$ is the number of samples generated after burn-in. Repeating the above sampling steps, we obtain a discrete-time Markov chain $\left\{\left(\rho^{(k)}, \sigma_{e}^{2(k)}\right) ; k=1,2, \ldots\right\}$ whose stationary distribution is the joint posterior density of the parameters.

The Gibbs sampling algorithm is performed via WinBUGS, then MCMC sample of $\theta_{2}=\left(\rho, \sigma_{e}^{2}\right)$ are obtained by computing mean of the joint posterior summaries.

\section{A simulation study}

The objective of this study is to estimate parameter $\theta_{1}=\left(\alpha, \mu_{\beta}, \sigma_{\beta}^{2}, \sigma_{\varepsilon}^{2}\right)^{\top}$ from $\operatorname{RCA}(1)$ and $\theta_{2}=$ $\left(\rho, \sigma_{e}^{2}\right)^{\top}$ from $\mathrm{RCA}(1)-\mathrm{AR}(1)$ by using the Bayesian analysis. The results have been shown to compare the average estimators in the sample sizes 100 and 500 . One of the most common measures is Mean Square Error (MSE), which is evaluated the difference between the estimated values and the real values. MSE measures the average of the square error, while the error becomes to be an amount by which the MSE can be compared to the quality estimated. We also computed the MSE as the criterion defined following:

$$
M S E=\frac{\sum_{t=1}^{n}\left(x_{t}-\hat{x}_{t}\right)^{2}}{n}
$$



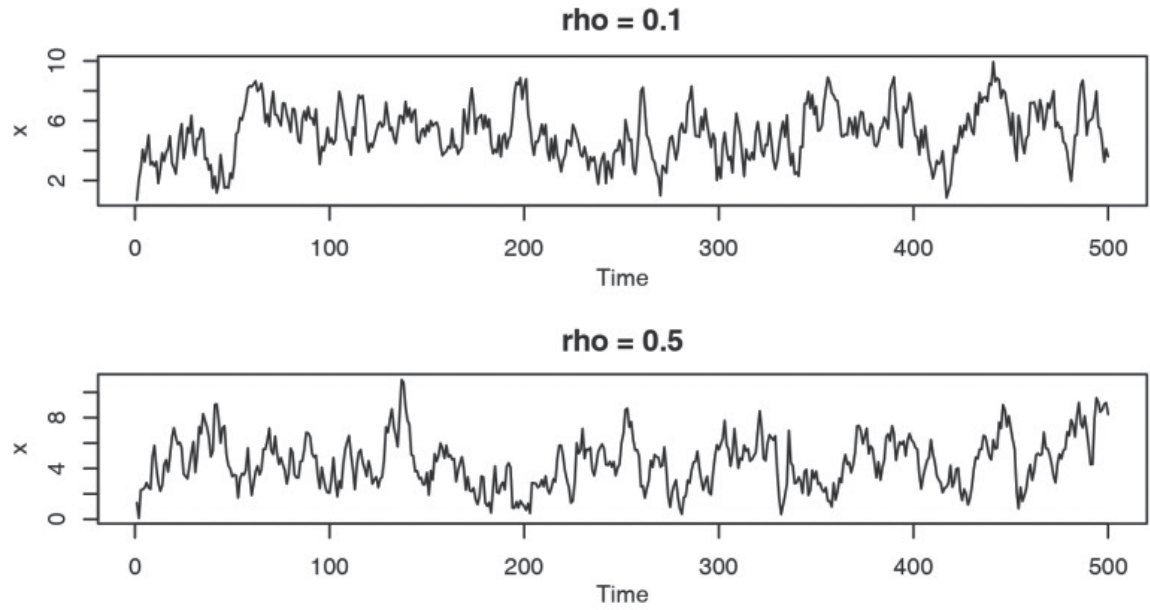

rho $=0.9$

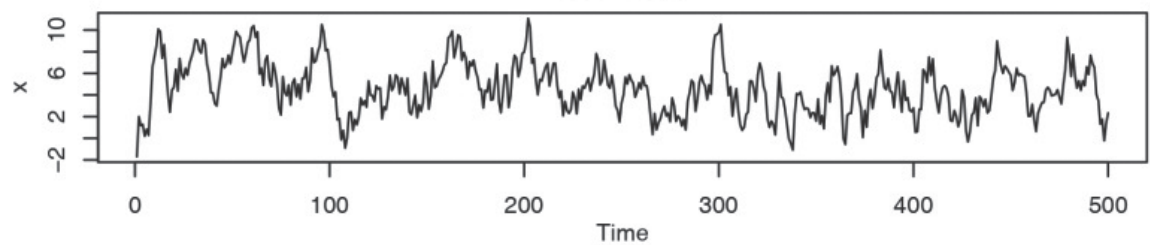

Fig. 1. The time series plot for generated data of $\operatorname{RCA}(1)-\operatorname{AR}(1)$ by fixing $\alpha=0, \sigma_{\varepsilon}^{2}=1, \sigma_{e}^{2}=1, \mu_{\beta}=0.8, \sigma_{\beta}^{2}=0$, and $\rho=0.1,0.5,0.9$. (500 sample sizes).
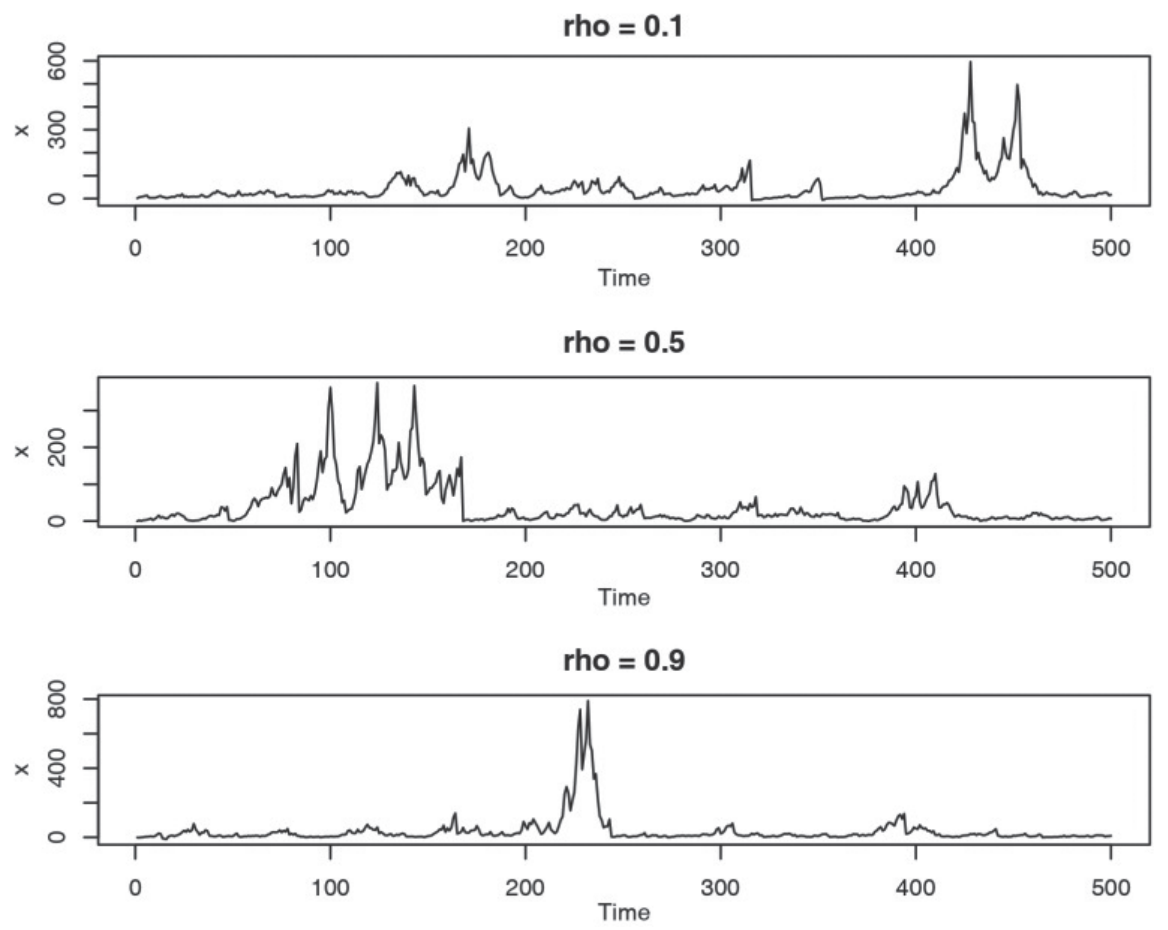

Fig. 2. The time series plot for generated data of $\operatorname{RCA}(1)-\operatorname{AR}(1)$ by fixing $\alpha=0, \sigma_{\varepsilon}^{2}=1, \sigma_{e}^{2}=1, \mu_{\beta}=0.995, \sigma_{\beta}^{2}=0.1$, and $\rho=0.1,0.5$, 0.9. (500 sample sizes). 
Table 1

Monte Carlo average and standard deviation of posterior mean, by fixing $\alpha=0, \sigma_{\varepsilon}^{2}=1$, $\sigma_{e}^{2}=1, \mu_{\beta}=0.8, \sigma_{\beta}^{2}=0$, and $\rho=0.1,0.5,0.9$ of RCA(1) and RCA(1)-AR(1) models

\begin{tabular}{lccccc}
\hline & \multicolumn{2}{c}{$n=100$} & & \multicolumn{2}{c}{$n=500$} \\
\cline { 2 - 3 } \cline { 5 - 6 } True & RCA $(1)$ & RCA(1)-AR(1) & & RCA $(1)$ & RCA(1)-AR(1) \\
\hline$\alpha=1$ & $1.02(0.44)$ & $1.02(0.44)$ & & $1.01(0.29)$ & $1.01(0.29)$ \\
$\mu_{\beta}=0.8$ & $0.04(0.20)$ & $0.04(0.20)$ & & $0.08(0.17)$ & $0.08(0.17)$ \\
$\sigma_{\beta}^{2}=0$ & $0.00(0.00)$ & $0.00(0.00)$ & & $0.00(0.00)$ & $0.00(0.00)$ \\
$\sigma_{\varepsilon}^{2}=1$ & $1.00(0.00)$ & $1.00(0.00)$ & & $1.00(0.00)$ & $1.00(0.00)$ \\
$\rho=0.1$ & - & $0.03(0.02)$ & & - & $0.01(0.01)$ \\
$\sigma_{e}^{2}=1$ & - & $0.99(0.00)$ & & - & $0.99(0.00)$ \\
AMSE & 21.07 & 19.83 & & 28.51 & 27.13 \\
$\alpha=1$ & $1.10(0.31)$ & $1.01(0.31)$ & & $1.03(0.14)$ & $1.03(0.14)$ \\
$\mu_{\beta}=0.8$ & $0.80(0.06)$ & $0.80(0.06)$ & & $0.04(0.02)$ & $0.04(0.02)$ \\
$\sigma_{\beta}^{2}=0$ & $0.00(0.00)$ & $0.00(0.00)$ & & $0.00(0.00)$ & $0.00(0.00)$ \\
$\sigma_{\varepsilon}^{2}=1$ & $1.00(0.00)$ & $1.00(0.00)$ & & $1.00(0.00)$ & $1.00(0.00)$ \\
$\rho=0.5$ & - & $0.08(0.03)$ & & - & $0.03(0.14)$ \\
$\sigma_{e}^{2}=1$ & - & $1.00(0.00)$ & & - & $1.00(0.00)$ \\
AMSE & 1.24 & 1.04 & & 1.27 & 1.18 \\
$\alpha=1$ & $1.11(0.34)$ & $1.11(0.34)$ & & $1.03(0.15)$ & $1.03(0.15)$ \\
$\mu_{\beta}^{2}=0.8$ & $0.80(0.06)$ & $0.80(0.06)$ & & $0.82(0.02)$ & $0.82(0.02)$ \\
$\sigma_{\beta}^{2}=0$ & $0.00(0.00)$ & $0.00(0.00)$ & & $0.00(0.00)$ & $0.00(0.00)$ \\
$\sigma_{\varepsilon}^{2}=1$ & $1.00(0.00)$ & $1.00(0.00)$ & & $1.00(0.00)$ & $1.00(0.00)$ \\
$\rho=0.9$ & - & $0.08(0.03)$ & & - & $0.03(0.01)$ \\
$\sigma_{e}^{2}=1$ & - & $1.00(0.00)$ & & - & $1.00(0.00)$ \\
AMSE & 1.79 & 1.51 & & 1.82 & 1.69 \\
\hline & & & & & \\
\hline
\end{tabular}

rho $=0.1$

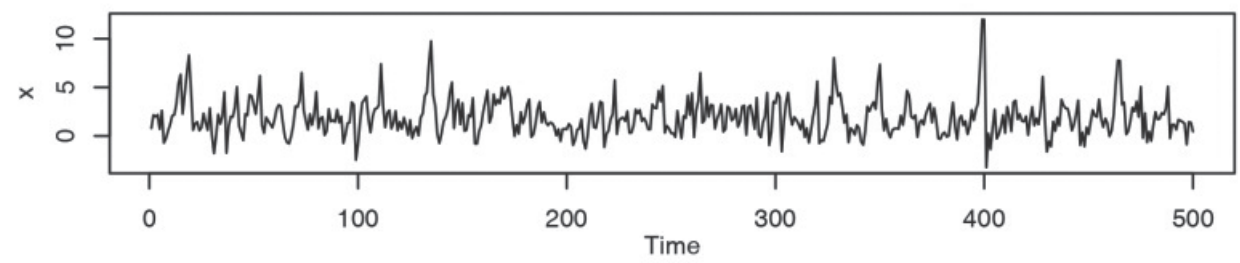

rho $=0.5$

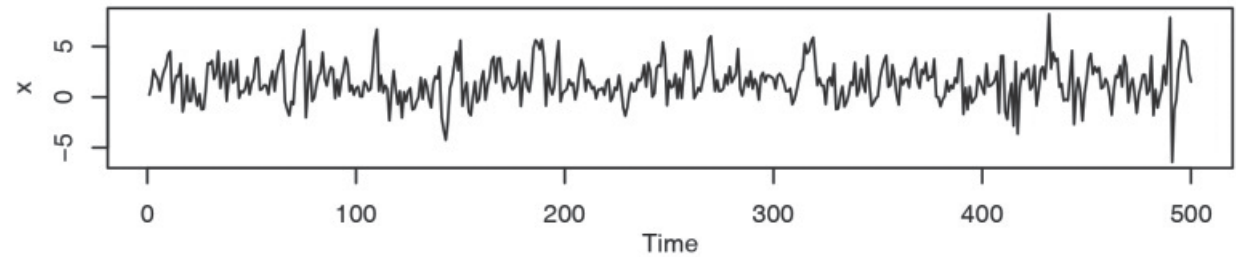

rho $=0.9$

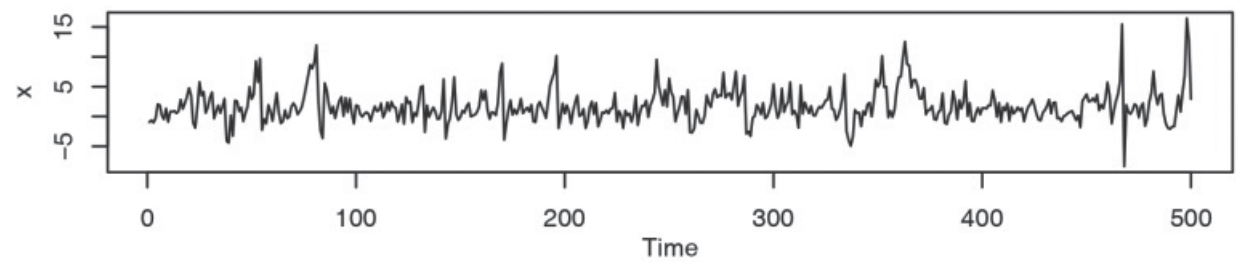

Fig. 3. The time series plot for generated data of $\operatorname{RCA}(1)-\operatorname{AR}(1)$ by fixing $\alpha=0, \sigma_{\varepsilon}^{2}=1, \sigma_{e}^{2}=1, \mu_{\beta}=0.5, \sigma_{\beta}^{2}=0.25$, and $\rho=0.1,0.5,0.9$. (500 sample sizes). 
Table 2

Monte Carlo average and standard deviation of posterior mean, by fixing $\alpha=0, \sigma_{\varepsilon}^{2}=1, \sigma_{e}^{2}=1$, $\mu_{\beta}=0.995, \sigma_{\beta}^{2}=0.1$, and $\rho=0.1,0.5,0.9$ of RCA(1) and RCA(1)-AR(1) models

\begin{tabular}{|c|c|c|c|c|}
\hline \multirow[b]{2}{*}{ True } & \multicolumn{2}{|c|}{$n=100$} & \multicolumn{2}{|c|}{$n=500$} \\
\hline & RCA(1) & RCA(1)-AR(1) & $\mathrm{RCA}(1)$ & $\mathrm{RCA}(1)-\mathrm{AR}(1)$ \\
\hline$\alpha=1$ & $1.12(0.29)$ & $1.12(0.29)$ & $1.03(0.14)$ & $1.03(0.14)$ \\
\hline$\mu_{\beta}=0.995$ & $0.76(0.05)$ & $0.76(0.05)$ & $0.79(0.02)$ & $0.79(0.02)$ \\
\hline$\sigma_{\beta}^{2}=0.1$ & $0.01(0.00)$ & $0.01(0.00)$ & $0.01(0.00)$ & $0.01(0.00)$ \\
\hline$\sigma_{\varepsilon}^{2}=1$ & $1.00(0.00)$ & $1.00(0.00)$ & $1.00(0.00)$ & $1.00(0.00)$ \\
\hline$\rho=0.1$ & - & $0.09(0.03)$ & - & $0.09(0.01)$ \\
\hline$\sigma_{e}^{2}=1$ & - & $0.98(0.00)$ & - & $0.98(0.00)$ \\
\hline AMSE & 0.98 & 0.81 & 1.00 & 0.92 \\
\hline$\alpha=1$ & 4.40(7.69) & 4.04(7.69) & $9.80(41.28)$ & $9.80(41.28)$ \\
\hline$\mu_{\beta}=0.995$ & $0.89(0.08)$ & $0.89(0.08)$ & $0.94(0.04)$ & $0.94(0.04)$ \\
\hline$\sigma_{\beta}^{2}=0.1$ & $0.01(0.00)$ & $0.01(0.00)$ & $0.01(0.00)$ & $0.01(0.00)$ \\
\hline$\sigma_{\varepsilon}^{2}=1$ & $1.00(0.00)$ & $1.00(0.00)$ & $1.00(0.00)$ & $1.00(0.00)$ \\
\hline$\rho=0.5$ & - & $0.09(0.07)$ & - & $0.05(0.06)$ \\
\hline$\sigma_{e}^{2}=1$ & - & $0.99(0.00)$ & - & $0.99(0.00)$ \\
\hline AMSE & 2866.80 & 2504.82 & 61689.40 & 49759.63 \\
\hline$\alpha=1$ & $5.16(14.23)$ & $5.16(14.23)$ & $6.59(14.30)$ & $6.59(14.30)$ \\
\hline$\mu_{\beta}=0.995$ & $0.86(0.09)$ & $0.86(0.09)$ & $0.90(0.04)$ & $0.90(0.04)$ \\
\hline$\sigma_{\beta}^{2}=0.1$ & $0.00(0.00)$ & $0.00(0.00)$ & $0.00(0.00)$ & $0.00(0.00)$ \\
\hline$\sigma_{\varepsilon}^{2}=1$ & $1.00(0.00)$ & $1.00(0.00)$ & $1.00(0.00)$ & $1.00(0.00)$ \\
\hline$\rho=0.9$ & - & $0.12(0.09)$ & - & $0.05(0.06)$ \\
\hline$\sigma_{e}^{2}=1$ & - & $0.99(0.00)$ & - & $0.99(0.00)$ \\
\hline AMSE & 12341.83 & 10821.51 & 73689.6 & 60893.54 \\
\hline
\end{tabular}

Table 3

Monte Carlo average and standard deviation of posterior mean, by fixing $\alpha=0, \sigma_{\varepsilon}^{2}=1, \sigma_{e}^{2}=$ $1, \mu_{\beta}=0.5, \sigma_{\beta}^{2}=0.25$, and $\rho=0.1,0.5,0.9$ of RCA(1) and RCA(1)-AR(1) models

\begin{tabular}{|c|c|c|c|c|}
\hline \multirow[b]{2}{*}{ true } & \multicolumn{2}{|c|}{$n=100$} & \multicolumn{2}{|c|}{$n=500$} \\
\hline & $\mathrm{RCA}(1)$ & $\mathrm{RCA}(1)-\mathrm{AR}(1)$ & $\mathrm{RCA}(1)$ & $\mathrm{RCA}(1)-\mathrm{AR}(1)$ \\
\hline$\alpha=1$ & $1.07(0.21)$ & $1.07(0.21)$ & $1.02(0.11)$ & $1.02(0.11)$ \\
\hline$\mu_{\beta}=0.5$ & $0.42(0.11)$ & $0.42(0.11)$ & $0.35(0.06)$ & $0.35(0.06)$ \\
\hline$\sigma_{\beta}^{2}=0.25$ & $0.21(0.13)$ & $0.21(0.13)$ & $0.24(0.05)$ & $0.24(0.05)$ \\
\hline$\sigma_{\varepsilon}^{2}=1$ & $1.00(0.00)$ & $1.00(0.00)$ & $1.00(0.00)$ & $1.00(0.00)$ \\
\hline$\rho=0.1$ & - & $0.81(0.05)$ & - & $0.85(0.05)$ \\
\hline$\sigma_{e}^{2}=1$ & - & $1.00(0.00)$ & - & $1.00(0.00)$ \\
\hline AMSE & 109.83 & 3.90 & 19.20 & 0.42 \\
\hline$\alpha=1$ & $1.07(0.23)$ & $1.07(0.23)$ & $1.03(0.13)$ & $1.03(0.13)$ \\
\hline$\mu_{\beta}=0.5$ & $0.32(0.20)$ & $0.32(0.20)$ & $0.24(0.06)$ & $0.24(0.06)$ \\
\hline$\sigma_{\beta}^{2}=0.25$ & $0.23(0.04)$ & $0.23(0.04)$ & $0.31(0.07)$ & $0.31(0.07)$ \\
\hline$\sigma_{\varepsilon}^{\beta}=1$ & $1.00(0.00)$ & $1.00(0.00)$ & $1.00(0.00)$ & $1.00(0.00)$ \\
\hline$\rho=0.5$ & - & $0.78(0.06)$ & - & $0.83(0.02)$ \\
\hline$\sigma_{e}^{2}=1$ & - & $1.00(0.00)$ & - & $1.00(0.00)$ \\
\hline AMSE & 128.55 & 6.301 & 23.82 & 0.69 \\
\hline$\alpha=1$ & $1.08(0.29)$ & $1.08(0.29)$ & $1.05(0.18)$ & $1.05(0.18)$ \\
\hline$\mu_{\beta}=0.5$ & $0.28(0.19)$ & $0.28(0.19)$ & $0.19(0.14)$ & $0.19(0.14)$ \\
\hline$\sigma_{\beta}^{2}=0.25$ & $0.25(0.08)$ & $0.25(0.08)$ & $0.39(0.09)$ & $0.39(0.09)$ \\
\hline$\sigma_{\varepsilon}^{2}=1$ & $1.00(0.00)$ & $1.00(0.00)$ & $1.00(0.00)$ & $1.00(0.00)$ \\
\hline$\rho=0.9$ & - & $0.71(0.08)$ & - & $0.79(0.03)$ \\
\hline$\sigma_{e}^{2}=1$ & - & $1.00(0.00)$ & - & $1.00(0.00)$ \\
\hline AMSE & 204.04 & 16.75 & 41.20 & 1.82 \\
\hline
\end{tabular}

where $x_{t}$ denotes the real values and $\hat{x}_{t}$ denotes the estimated values.

In this case, simulation study is divided in three parts.

At the first, we generate data $x_{t}, t=1,2, \ldots, n$ from
$\operatorname{RCA}(1)-\mathrm{AR}(1)$ by taking $\alpha=0, \sigma_{\varepsilon}^{2}=1, \sigma_{e}^{2}=1$, and $\rho=0.1,0.5$ and 0.9 following

$$
x_{t}=\alpha+\mu_{\beta} x_{t-1}+\rho \varepsilon_{t-1}+e_{t} .
$$

To illustrate the implication of RCA(1)-AR(1) mo- 
del, Figs 1-3 show generating data of 500 sample sizes and $\rho=0.1,0.5$ and 0.9 for each 3 cases as;

1. $\mu_{\beta}=0.8, \sigma_{\beta}^{2}=0$

2. $\mu_{\beta}=0.995, \sigma_{\beta}^{2}=0.1$

3. $\mu_{\beta}=0.5, \sigma_{\beta}^{2}=0.25$.

It should be noted that case 1 is the stationary process, case 2 is displayed the nonstationarity or random walk case, and case 3 also tends to oscillate around its mean zero.

In the second, we obtain the estimator $\hat{\theta}_{1}=$ $\left(\hat{\alpha}, \hat{\mu}_{\beta}, \hat{\sigma}_{\beta}^{2}, \hat{\sigma}_{\varepsilon}^{2}\right)^{\top}$ from MCMC method and we get $x_{t}$ from

$$
x_{t}=\hat{\alpha}+\hat{\mu}_{\beta} x_{t-1}, t=2,3, \ldots, n .
$$

Let

$$
\hat{\varepsilon}_{t}=x_{t}-\left(\hat{\alpha}+\hat{\mu}_{\beta} x_{t-1}\right), t=2,3, \ldots, n,
$$

the MCMC method is used to estimate parameter $\hat{\theta}_{2}=$ $\left(\hat{\rho}, \hat{\sigma}_{e}^{2}\right)^{\top}$. It's become

$$
x_{t}=\hat{\alpha}+\hat{\mu}_{\beta} x_{t-1}+\hat{\rho} \hat{\varepsilon}_{t-1}, t=2,3, \ldots, n .
$$

Finally, in the third part, we simulate data at 500 replications from RCA(1)-AR(1) model. We also compute the Monte Carlo averages and standard deviation of the posterior mean for each parameter based on sample size such as $n=100$ and $n=500$. To see this we compute the Average of MSE (AMSE) for comparing the effective estimation between $\mathrm{RCA}(1)$ and $\mathrm{RCA}(1)$ AR(1) models. If the AMSEs have a lower value, these models will fit to estimation.

From Tables 1-3, we also list the average standard deviation of the posterior mean for fitting $\mathrm{RCA}(1)$ and RCA(1)-AR(1) models. The results appear that the average of posterior mean seems to decrease with increasing sample sizes. For the AMSE, the RCA(1)$\mathrm{AR}(1)$ model performs better than $\mathrm{RCA}(1)$ model in all cases, since the correlation error process occurs on the generating data, so the correlation is adjusted by RCA(1)-AR(1) model.

\section{Applications in real data}

In this section, we consider the application of RCA(1) and RCA(1)-AR(1) models using the Bayesian approach that we developed in the previous section and shown the output at Table 4 and Fig. 4. The real data, we use the monthly volume of the Stock Exchange
Table 4

Posterior summaries (average and standard deviation) of RCA(1) and RCA(1)-AR(1) estimator of SET index

\begin{tabular}{ccc}
\hline Parameters & $\mathrm{RCA}(1)$ & $\mathrm{RCA}(1)-\mathrm{AR}(1)$ \\
\hline$\alpha$ & $6.62(4.50)$ & $6.62(4.50)$ \\
$\mu_{\beta}$ & $0.31(434.76)$ & $0.31(434.76)$ \\
$\sigma_{\beta}^{2}$ & $102.11(5.78)$ & $102.11(5.78)$ \\
$\sigma_{\varepsilon}^{2}$ & $3058.80(21.30)$ & $3058.80(21.30)$ \\
$\rho$ & - & $0.99(0.004)$ \\
$\sigma_{e}^{2}$ & - & $3251.15(220.98)$ \\
$\mathrm{MSE}$ & 198124.8 & 13.163 \\
\hline
\end{tabular}

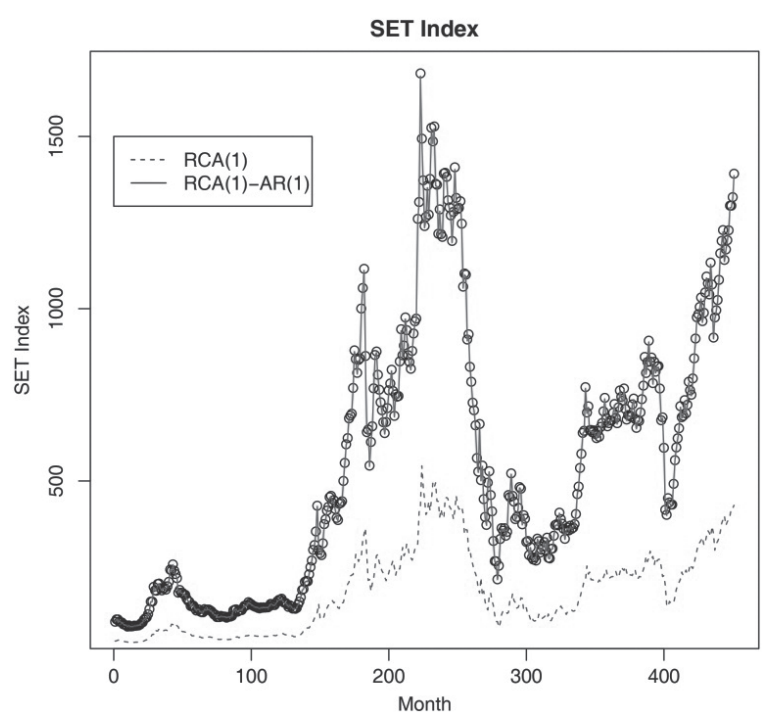

Fig. 4. The time series plot for SET index and estimated of RCA(1), AR(1) model.

of Thailand (SET) index that is started for trading on April 30, 1975. This data are collected from 1975 to 2012 giving a total of 453 observations which is collected from http://www.set.or.th/th/market/market statistics.html.

From Table 4, the MSE of the RCA(1)-AR(1) model are smaller than the RCA(1) model, so the RCA(1)AR(1) model performs to estimate SET index better than the RCA(1) model.

In Fig. 4, the bottom panel is the plot of SET index, the dashed line is the RCA(1) estimated model, and the line is the RCA(1)-AR(1) estimated model. It is seen that the RCA(1)-AR(1) model are competitive model for fitting SET index.

\section{Conclusion}

In this paper, we have developed the Bayesian analysis for estimating parameters of the first order in RCA 
and RCA-AR models which are called the RCA(1) and RCA(1)-AR(1) models. For the simulation study, we also investigated the performance of Bayesian analysis and showed the averages and standard deviation of the posterior mean, and AMSEs for different data at the sample size of 100 and 500. It appears that all cases of $\mathrm{RCA}(1)-\mathrm{AR}(1)$ model performs well in picking up the correct model, to see the AMSE is minimum. It is indicated that it is the time series data based on the errors which are affected the estimation.

For application in real data, we are also interested the power of estimating by considering the Mean Square Error (MSE). We can see that the RCA(1)AR(1) model performs better than the RCA(1) as seen from MSE of the RCA(1)-AR(1) model. It is smaller than the MSE of the RCA(1) model. We would recommend users to fit the RCA(1)-AR(1) models to time series data where stationary and non-stationary data are expected.

\section{References}

[1] R.F. Engle, Autoregressive conditional heteroscedasticity with estimates of the variance of United.

[2] T. Bollerslev, Generalized autoregressive conditional heteroscedasticity, Journal of Econometrics 31 (1986), 307-327.
[3] R. Tsay, Conditional heteroscedastic time series models, Journal of the American Statistical Association 82 (1987), 590-604.

[4] D.F. Nicholls and B.G. Quinn, Random coefficient autoregressive models: An introduction Springer-Verlag Inc, New York, 1982.

[5] S.Y. Hwang and I.V. Basawa, Parameter estimation for generalized random coefficient autoregressive process, Journal of Statistical Planning and Inference 68 (1998), 323-337.

[6] S.A. Chandra and M. Taniguchi, Estimating Functions for Nonlinear Time Series Data, Annals of the Institute of Statistical Mathematics 53 (2001), 125-136.

[7] D. Wang and S.K. Ghosh, Bayesian analysis of random coefficient autoregressive models, Model Assisted Statistics and Applications 3 (2002), 281-295.

[8] V. Haggan and T. Ozaki, Modeling nonlinear vibrations using an amplitude-dependent autoregressive time series model, Biometrika 68 (1981), 189-196.

[9] H. Tong, Threshold models in nonlinear time series analysis, Springer-Verlag Inc, Heidelberg, 1982.

[10] T. Bossaerts, W. Hardle and C. Hafner, Foreign exchangerates have surprising volatility, Athens Conference on Applied Probability and Time Series 2 (1996), 55-72.

[11] A. Gelfand and A.F.M. Smith, Sampling-based approaches to calculating marginal densities, Journal of American Statistical Association 85 (1990), 398-409.

[12] S. Geman and D. Geman, Stochastic relaxation, Gibbs distributions and the Bayesian restoration of images, IEEE Transactions on Pattern Analysis and Machine Intelligence 6 (1984), 721-741.

[13] D.J. Lunn, A. Thomas, N. Best and D. Spiegelhalter, WinBUGS-a Bayesian modeling frameork: Concepts, structure, and extensibility, Statistics and Computing 10 (2000), 325-337. 